\title{
A SIMPLE ROBUST PID CONTROLLER DESIGN METHOD BASED ON SINE WAVE IDENTIFICATION OF THE UNCERTAIN PLANT
}

\author{
Štefan Bucz - Ladislav Marič \\ Ladislav Harsányi — Vojtech Veselý
}

\begin{abstract}
The paper deals with the development and application of a new simple empirical approach to the design of robust PID controllers for technological processes in industrial practice. The main advantage of the proposed approach is the possibility to specify the required performance before the design algorithm implementation. Identification of characteristic data of the black-box type plant with varying parameters is carried out using the sine wave excitation signal, thus allowing to design the controller without necessarily knowing the mathematical model of the plant. The proposed approach has been verified on a real-world physical process.
\end{abstract}

K e y w or d s: empirical methods, characteristic data of the plant, excitation signal, robust stability, robust performance

\section{INTRODUCTION}

Empirical methods are popular tools to design industrial controllers [9]. Usually, the design proceeds in twosteps: the first step is identification of certain characteristic information about the controlled plant; the second step is the controller design with parameters that directly depend on the identified data. The broad use of these methods is due to their algorithm simplicity and quick controller synthesis as well as to minimum of a priori information about the plant necessary for a successful design [11].

Nevertheless, quite frequently controllers implemented in various industrial technologies are not tuned effectively thus bringing about economic losses. The existing studies have revealed that certain controllers not only do not guarantee the required closed-loop performance but even fail to provide closed-loop stability or are operated just in an open-loop and on achieving setpoint vicinity they are manually switched-off or are further operated by the operation staff [4].

The main advantage of the proposed empirical design method is a quick design of a robust PID controller for specified performance without knowledge of an exact mathematical model of the plant. Characteristic plant data are obtained by performing a small number of experiments on the plant during the design process [1].

\section{CONTROL LAW OF THE EMPIRICAL SINE WAVE METHOD}

For controller design purpose, stability and performance are usually specified in terms of stability margins
[2]. The proposed empirical design method enables the required phase margin $\phi_{M}$ to be specified by the control engineer or process technologist. The control law is easy to derive from the closed-loop characteristic equation of the control loop in Fig. 1 when the switch SB is in position " 1 ".

In Fig. 1, $G(s)$ is the plant transfer function with an unknown mathematical model and $G_{R}(s)$ is the transfer function of the industrial PID controller. From the closedloop transfer function

$$
T(j \omega)=\frac{L(j \omega)}{1+L(j \omega)}=\frac{G(j \omega) G_{R}(j \omega)}{1+G(j \omega) G_{R}(j \omega)}
$$

results the closed-loop characteristic equation

$$
1+L\left(j \omega_{n}\right)=1+G\left(j \omega_{n}\right) G_{R}\left(j \omega_{n}\right)=0
$$

which can be easily broken down into the magnitude and phase conditions

$$
\begin{gathered}
\left|G\left(j \omega_{n}\right)\right|\left|G_{R}\left(j \omega_{n}\right)\right|=1, \\
\arg G\left(\omega_{n}\right)+\arg G_{R}\left(\omega_{n}\right)=-\pi+\phi_{M},
\end{gathered}
$$

where $\phi_{M}$ is the required phase margin specified in advance, $L(j \omega)$ is the open-loop transfer function. Introduce the following substitution

$$
\varphi=\arg G\left(\omega_{n}\right), \Theta=\arg G_{R}\left(\omega_{n}\right) .
$$

Consider a PID controller

$$
G_{R}(s)=K\left[1+\frac{1}{s T_{i}}+s T_{d}\right]
$$

\footnotetext{
* Institute of Control and Industrial Informatics, Slovak University of Technology in Bratislava, Faculty of Electrical Engineering and Information Technology, Ilkovičova 3, 81219 Bratislava, Slovakia, bucz@tracon.sk, lharsanyi@elf.stuba.sk, vojtech.vesely@stuba.sk
} 


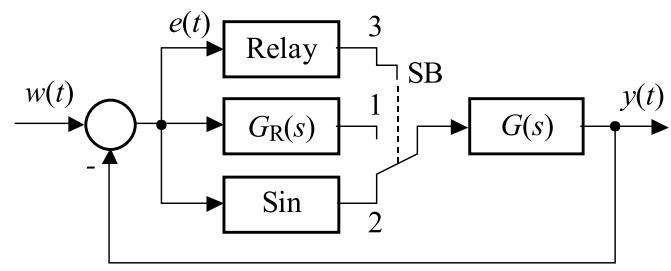

Fig. 1. Feedback loop for the sine wave experimental method

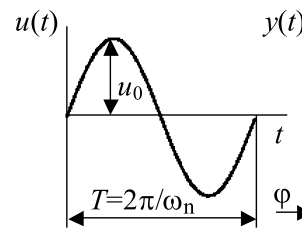

(a)

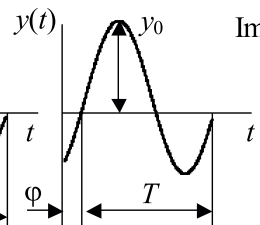

(b)

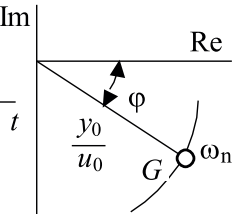

(c)
Fig. 2. Identification procedure of one point of the plant frequency response using the sine wave method

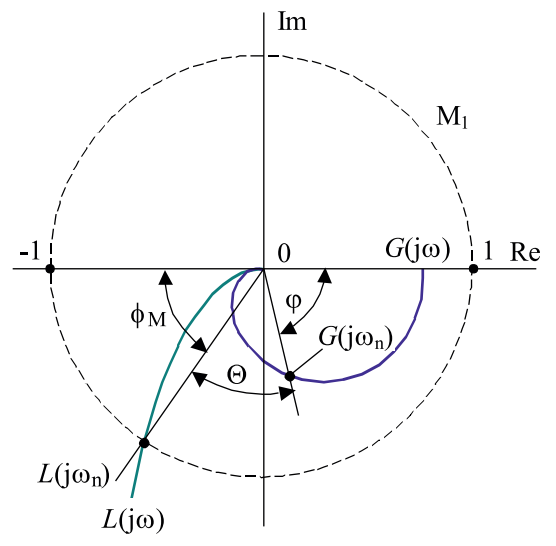

Fig. 3. Mutual position of $G\left(j \omega_{n}\right)$ and $L\left(j \omega_{n}\right)$ and verification of the phase condition (23) in the complex plane

where $K$ is the proportional gain, and $T_{i}$ and $T_{d}$ are integral and derivative time constants, respectively. A frequency domain comparison of the right-hand side of (5)

$$
G_{R}\left(j \omega_{n}\right)=K+j K\left[T_{d} \omega_{n}-\frac{1}{T_{i} \omega_{n}}\right]
$$

with the right-hand side of the PID in polar form

$$
\begin{aligned}
& G_{R}\left(j \omega_{n}\right)=\left|G_{R}\left(j \omega_{n}\right)\right| e^{j \Theta}= \\
& \left|G_{R}\left(j \omega_{n}\right)\right| \cos \Theta+j\left|G_{R}\left(j \omega_{n}\right)\right| \sin \Theta
\end{aligned}
$$

yields a complex equality

$$
K+j K\left[T_{d} \omega_{n}-\frac{1}{T_{i} \omega_{n}}\right]=\frac{\cos \Theta}{\left|G\left(j \omega_{n}\right)\right|}+j \frac{\sin \Theta}{\left|G\left(j \omega_{n}\right)\right|}
$$

from which it is possible to obtain PID controller parameters using the substitution

$$
\left|G_{R}\left(j \omega_{n}\right)\right|=\frac{1}{\left|G\left(j \omega_{n}\right)\right|}
$$

resulting from the magnitude condition (3a).

The controller gain $K$ can be expressed directly from the complex equation (8)

$$
K=\frac{\cos \Theta}{\left|G\left(j \omega_{n}\right)\right|}
$$

and the derivative time constant $T_{d}$ can be specified from the quadratic equation with respect to $T_{d} \omega_{n}$

$$
K\left[T_{d} \omega_{n}-\frac{1}{\beta T_{d} \omega_{n}}\right]=\frac{\sin \Theta}{\left|G\left(j \omega_{n}\right)\right|} .
$$

The ratio of integral and derivative time constants is set by an appropriate choice of coefficient $\beta$ (according to the most frequently used empirical methods $\beta=4$ [9])

$$
\beta=\frac{T_{i}}{T_{d}} \Longrightarrow T_{i}=\beta T_{d}
$$

Substituting (10) into (11) yields a quadratic equation with respect to $T_{d} \omega_{n}$

$$
\left[T_{d} \omega_{n}-\frac{1}{\beta T_{d} \omega_{n}}\right]=\operatorname{tg} \Theta
$$

that can simply be modified as follows

$$
T_{d}^{2} \omega_{n}^{2}-T_{d} \omega_{n} \operatorname{tg} \Theta-\frac{1}{\beta}=0 .
$$

From the solution of (14) directly results an expression for calculation of the derivative time constant $T_{d}$

$$
T_{d}=\frac{\operatorname{tg} \Theta}{2 \omega_{n}} \pm \frac{1}{\omega_{n}} \sqrt{\frac{\operatorname{tg}^{2} \Theta}{4}+\frac{1}{\beta}} .
$$

Hence, PID controller parameters are calculated according to the following expressions

$$
\begin{gathered}
K=\frac{\cos \Theta}{\left|G\left(j \omega_{n}\right)\right|}, T_{i}=\beta T_{d}, \\
T_{d}=\frac{\operatorname{tg} \Theta}{2 \omega_{n}} \pm \frac{1}{\omega_{n}} \sqrt{\frac{\operatorname{tg}^{2} \Theta}{4}+\frac{1}{\beta}},
\end{gathered}
$$

where angle $\Theta$ is obtained from the phase condition (3b)

$$
\Theta=-\pi+\Phi_{M}-\arg G\left(\omega_{n}\right)
$$




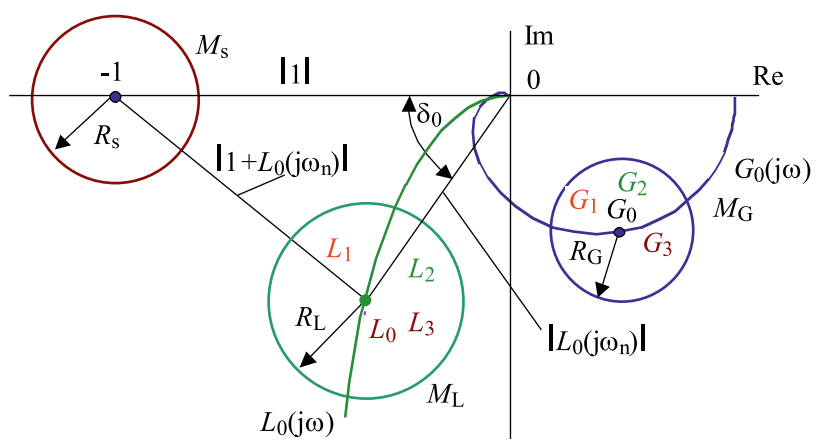

Fig. 4. Dispersion circles $M_{G}, M_{L}$ and the prohibited area delimited by the circle $M_{s}$

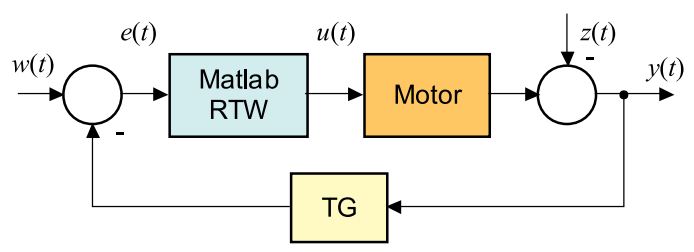

Fig. 5. Control loop of the DC motor with uncertainties

\section{IDENTIFICATION OF THE SINGLE POINT OF THE PLANT FREQUENCY RESPONSE USING THE SINE WAVE METHOD}

The proposed PID controller synthesis is based on the knowledge of a single point of the frequency response $G \equiv G\left(j \omega_{n}\right)$ of the unknown plant

$$
G\left(j \omega_{n}\right)=\left|G\left(j \omega_{n}\right)\right| e^{j \varphi\left(\omega_{n}\right)}
$$

If the reference variable in the control loop in Fig. 1 equals zero, ie $w(t)=0$, and the switch SB is in position " 2 , the control variable injected into the unknown plant $G(s)$ is generated by a sine wave signal

$$
u(t)=U_{0} \sin \left(\omega_{n} t\right)
$$

where $U_{0}$ is the amplitude of the excitation signal $u(t)$ and $\omega_{n}$ is the excitation frequency of the sine wave generator. The output signal $y(t)$ is also of sine wave type with the same frequency and amplitude $Y_{0}$

$$
y(t)=Y_{0} \sin \left(\omega_{n} t+\varphi\right)
$$

$\varphi$ is the phase shift with respect to the excitation signal $u(t)$. The corresponding time response is depicted in Fig. 2b. After reading off the values $Y_{0}$ and $\varphi$ from the recorded output, the particular point of the plant frequency characteristics corresponding to the excitation frequency $\omega_{n}$

$$
G\left(j \omega_{n}\right)=\frac{Y_{0}}{U_{0}} e^{j \varphi\left(\omega_{n}\right)}
$$

is plotted in the complex plane as depicted in Fig. 2c.

The advantage of the sine wave identification is that the sine signal amplitude $Y_{0}$ of the output $y(t)$ can be affected by the amplitude $U_{0}$ of the excitation sine signal $\mathrm{u}(\mathrm{t})$ generated by the sine wave generator.

Substituting coordinates of the identified point of the frequency response of the unknown plant $G$ (21) into (16a) yields final expressions for PID controller parameters

$$
\begin{gathered}
K=\frac{U_{0}}{Y_{0}} \cos \Theta, T_{i}=\beta T_{d}, \\
T_{d}=\frac{\operatorname{tg} \Theta}{2 \omega_{n}} \pm \frac{1}{\omega_{n}} \sqrt{\frac{\operatorname{tg}^{2} \Theta}{4}+\frac{1}{\beta}},
\end{gathered}
$$

where for the particular excitation frequency $\omega_{n}$ the following phase condition holds

$$
\Theta=-\pi+\Phi_{M}-\varphi
$$

The identified point $G$ of the plant frequency response $G(j \omega)$

$$
G=G\left(j \omega_{n}\right)=\left[\left|G\left(j \omega_{n}\right)\right|, \arg G\left(j \omega_{n}\right)\right]=\left[\left|G\left(j \omega_{n}\right)\right|, \varphi\right]
$$

determines the amplitude crossover $L$ of the open-loop $L(j \omega)$

$$
L=L\left(j \omega_{n}\right)=\left[\left|L\left(j \omega_{n}\right)\right|, \arg L\left(\omega_{n}\right)\right]=\left[\left|L\left(j \omega_{n}\right)\right|, \Phi_{M}\right]
$$

in which the designed PID controller guarantees the required phase margin $\Phi_{M}$. Therefore under the excitation frequency $\omega_{n}$ it holds $\left|L\left(j \omega_{n}\right)\right|=1$. Mutual position of the points $G\left(j \omega_{n}\right)$ and $L\left(j \omega_{n}\right)$ is depicted in Fig. 3 .

The empirical sine wave method evolves from the fulfilment of the phase condition (23) for the angular frequency $\omega_{n}$ of the identified point $G$ and simultaneously of the amplitude crossover of $L(j \omega)$. Similarly as a PID controller designed using Ziegler-Nichols tuning rules [1] compensating the critical point of the plant $G(j \omega c)$ by shifting it into the open-loop point $C_{P I D}=$ $[-0.6,-j 0.28]$, the PID controller tuned according to the empirical sine wave method moves the identified point $G\left(j \omega_{n}\right)$ of the plant frequency response into the openloop amplitude crossover $L\left(j \omega_{n}\right)$ lying on the unit circle $M_{1}$.

Note that in general a critical point of any plant is a point of its frequency characteristics with a phase shift $\varphi=-180^{\circ}$, where $\omega_{c}$ is the critical frequency of the plant.

It is advantageous to derive the frequency of the sine wave generator from the plant critical frequency $\omega_{c}$ that can be determined by the relay experiment according Rotač [8]. The experiment is carried out by switching the switch in the block diagram in Fig. 1 to the position " 3 ". The sine wave generator frequency is chosen from the interval

$$
\omega_{n} \in\left\langle 0.3 \omega_{c}, \omega_{c}\right\rangle \text {. }
$$

As the choice of the frequency $\omega_{n}$ influences the closed- loop dynamics, the interval (26) enables to modify the closed- loop dynamics [10]. 




$t(\mathrm{~s})$
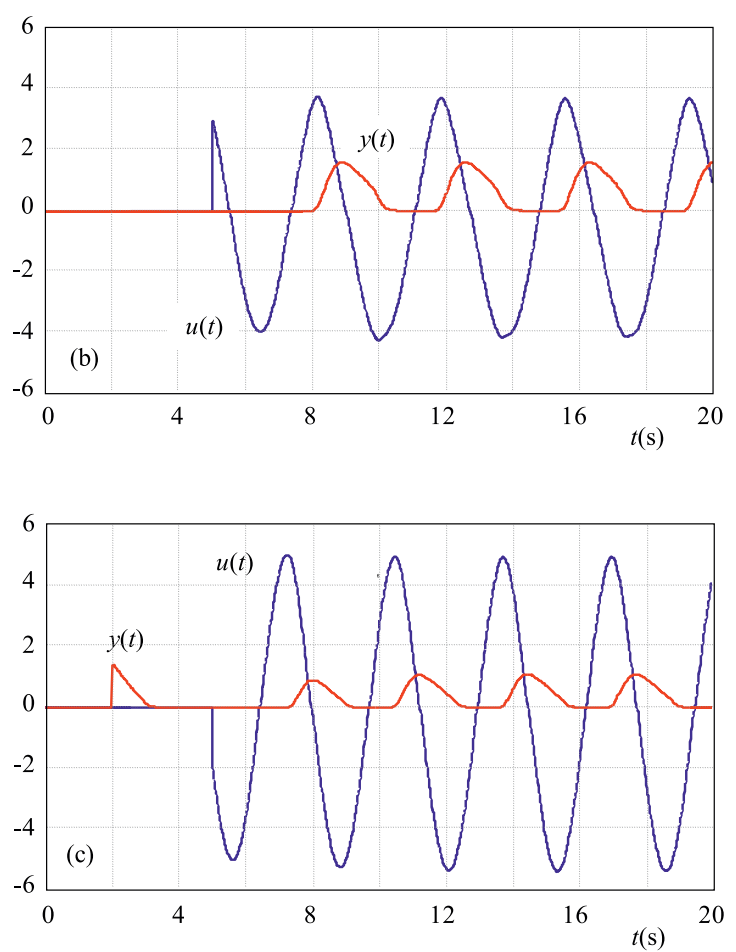

Fig. 6. Identification of DC motor in three working points using the sine wave method (for $Z_{\min }, Z_{\text {med }}, Z_{\max }$ )

\section{IDENTIFICATION OF THE UNCERTAIN PLANT AND DESIGN OF ROBUST PID CONTROLLER}

If the plant model is uncertain (due to variations in humidity, ambient temperature, linearization etc), it cannot be represented by a single frequency characteristics but by a set of frequency characteristics. The basic idea of identification of such an uncertain system consists in repeating the sine wave identification at the excitation signal frequency $\omega_{n}$ for individual uncertainty changes yielding a set of identified points $G_{i}, i=1,2, \ldots, N$ of the uncertain plant frequency responses

$$
G_{i}\left(j \omega_{n}\right)=\left|G_{i}\left(j \omega_{n}\right)\right| e^{j \arg G_{i}\left(\omega_{n}\right)}
$$

Plant parameter changes are reflected in magnitude and phase changes $\left|G_{i}\left(j \omega_{n}\right)\right|$ and $\arg G_{i}\left(\omega_{n}\right)$, respectively, of the identified points $G_{i}\left(j \omega_{n}\right)$ in the complex plane.

If the multiple identification of individual points $G_{i}$ of the uncertain plant frequency characteristics is performed using sine wave excitation signals with the same frequency $\omega_{n}$ in each identification experiment, then each identified point $G_{i}$ in the complex plane corresponds to a different frequency characteristics from the set of plant models and simultaneously, each identified point $G_{i}$ in the complex plane corresponds to the same angular frequency $\omega_{n}$ for $i=1,2, \ldots, N$; where $N=p+1$ is the number of identification experiments and $p$ is the number of varying technological quantities of the plant [6].

Location of identified points $G_{i}\left(j \omega_{n}\right)$ of the unknown uncertain plant can be expressed in the complex number standard form

$$
G_{i}\left(j \omega_{n}\right)=a_{i}+j b_{i}, i=1,2, \ldots, N .
$$

The real and imaginary parts of the nominal plant model $G_{0}\left(j \omega_{n}\right)$

$$
G_{0}\left(j \omega_{n}\right)=a_{0}+j b_{0}
$$

can be obtained as mean values of real and imaginary parts of identified points according to

$$
G_{0}\left(j \omega_{n}\right)=a_{0}+j b_{0}=\frac{1}{N} \sum_{i=1}^{N} a_{i}+j \frac{1}{N} \sum_{i=1}^{N} b_{i} .
$$

The nominal point $G_{0}\left(j \omega_{n}\right)$ expressed in polar form is

$$
G_{0}\left(j \omega_{n}\right)=\left|G_{0}\left(j \omega_{n}\right)\right| e^{j \varphi_{0}\left(\omega_{n}\right)},
$$

where the magnitude $\left|G_{0}\left(j \omega_{n}\right)\right|$ and the phase $\varphi_{0}\left(\omega_{n}\right)=$ $\arg G_{0}\left(j \omega_{n}\right)$ are calculated as follows

$$
\left|G_{0}\left(j \omega_{n}\right)\right|=\sqrt{a_{0}^{2}+b_{0}^{2}}, \varphi_{0}\left(\omega_{n}\right)=\operatorname{arctg} \frac{b_{0}}{a_{0}} .
$$

The points $G_{i}$ representing plant uncertainties can be enclosed in the circle $M_{G}$ centred in $G_{0}\left(j \omega_{n}\right)$ with the radius $R_{G} \equiv R_{G}\left(\omega_{n}\right)$ that can be obtained as a maximum distance between the $i$-th identified point $G_{i}\left(j \omega_{n}\right)$ and the nominal point $G_{0}\left(j \omega_{n}\right)$ according to

$$
\begin{aligned}
R_{G} & =\max \left\{\sqrt{\left(a_{i}-a_{0}\right)^{2}+\left(b_{i}-b_{0}\right)^{2}}\right\}, \\
i & =1,2, \ldots, N .
\end{aligned}
$$

The dispersion circle $M_{G}$ centred in the nominal point $G_{0}$ with the radius $R_{G}$ encircles all identified points $G_{i}$ of the uncertain plant. Figure 4 illustrates the situation for $N=3$ identifications.

The proposed control law generated by the robust controller $G_{\text {Rrob }}(s)$ designed for the nominal point $G_{0}\left(j \omega_{n}\right)$ actually carries out the transformation

$$
\Re:\left\{R_{G} \rightarrow R_{L}: R_{L}=\left|G_{\mathrm{Rrob}}\right| R_{G}\right\}
$$

of the set of identified points $G_{i}\left(j \omega_{n}\right)$ encircled by $M_{G}$ with the radius $R_{G}$ into the set of points $L_{i}\left(j \omega_{n}\right)$ delimited by $M_{L}$ and also calculates the radius $R_{L} \equiv R_{L}\left(\omega_{n}\right)$ of the dispersion circle $M_{L}$ corresponding to the points $L_{i}\left(j \omega_{n}\right)$ of the Nyquist plot so as to guarantee fulfilment of the robust stability condition. 

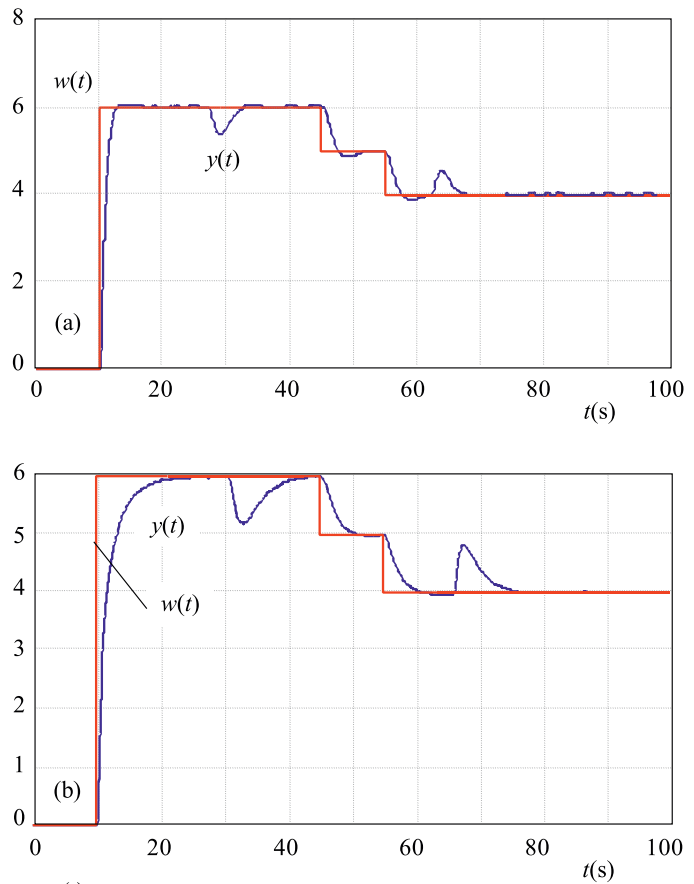

$u(t)$

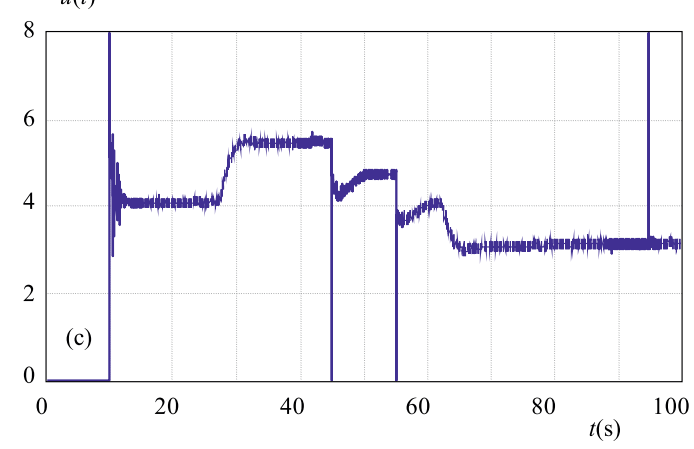

Fig. 7. DC motor closed-loop time responses for different $\phi_{M}$

Table 1. Robust PID controller coefficients for various $\phi_{M}$

\begin{tabular}{cccccc}
\hline $\begin{array}{c}\text { Controller } \\
\text { type }\end{array}$ & $\phi_{M}$ & $K$ & $T_{i}$ & $T_{d}$ & $\delta_{0}$ \\
\hline PID & $50^{\circ}$ & 1.1883 & 0.3448 & 1.3793 & $50^{\circ}$ \\
PID & $70^{\circ}$ & 0.8236 & 0.4754 & 1.9018 & $70^{\circ}$ \\
\hline
\end{tabular}

The robust PID controller is designed using the empirical sine wave method; the input data for the nominal model $G_{0}\left(j \omega_{n}\right)$ are its following coordinates

$$
A_{0}=\left|G_{0}\left(j \omega_{n}\right)\right|, \varphi_{0}=\arg G_{0}\left(\omega_{n}\right) .
$$

Substituting coordinates of the nominal model $G_{0}\left(j \omega_{n}\right)$ into (22) following expressions for calculating robust PID controller parameters are obtained

$$
\begin{gathered}
K_{\mathrm{rob}}=\frac{\cos \Theta_{0}}{A_{0}}, T_{i_{\mathrm{rob}}}=\beta T_{d_{\mathrm{rob}}} \\
T_{d_{\mathrm{rob}}}=\frac{\operatorname{tg} \Theta_{0}}{2 \omega_{n}} \pm \frac{1}{\omega_{n}} \sqrt{\frac{\operatorname{tg}^{2} \Theta_{0}}{4}+\frac{1}{\beta}}
\end{gathered}
$$

For the parameter $\Theta_{0}$ and excitation frequency $\omega_{n}$ the modified phase condition holds

$$
\Theta_{0}=-\pi+\delta_{0}-\varphi_{0} .
$$

Thus, $\delta_{0}$ is a modified phase margin and at the same time a robust PID controller tuning parameter appearing in (36) and (37) for calculation of its parameters that guarantee the necessary phase margin required for robust stability; $\delta_{0}$ does not influence the radius of the dispersion circle $M_{L}$, just the distance between $L_{0}$ and the critical point $(-1,0)$. This enables to draw the circle $M_{L}$ apart from the critical point $(-1,0)$ thus improving robust closed-loop performance.

\section{ROBUST STABILITY CONDITION}

If the nominal open-loop

$$
L_{0}(s)=G_{R}(s) G_{0}(s) \text {, }
$$

is stable, then according to the Nyquist stability criterion [7] the closed-loop with uncertain system will be stable if the distance between $L_{0}$ and the point $\left(-1, j_{0}\right)$, ie $\left|1+L_{0}\left(j \omega_{n}\right)\right|$, will be greater than the radius $R_{L}\left(\omega_{n}\right)$ of the dispersion circle $M_{L}$ centred in $L_{0}$ [3], [6]

$$
R_{L}\left(\omega_{n}\right)<\left|1+L_{0}\left(j \omega_{n}\right)\right|
$$

where $\omega_{n}$ is the frequency of the sine wave generator. The distance between the point $(-1,0)$ and the openloop Nyquist plot with the nominal model $L_{0}$ can be calculated according to Fig. 3 by applying the cosine rule to the triangle $\left(-1,0, L_{0}\right)$

$$
\left|1+L_{0}\right|^{2}=|1|^{2}+\left|L_{0}\right|^{2}-2 \cdot 1 \cdot\left|L_{0}\right| \cos \delta_{0},
$$

where $\delta_{0}$ is the modified phase margin.

According to the robust stability condition the distance

$$
\left|1+L_{0}\right|=\sqrt{1+\left|L_{0}\right|^{2}-2\left|L_{0}\right| \cos \delta_{0}},
$$

has to be greater than the radius $R_{L}$ of the dispersion circle centred in $L_{0}$, ie the following inequality has to be satisfied

$$
R_{L} \stackrel{!}{<}\left|1+L_{0}\right|
$$

Substituting the distance (41) into (42) yields the robust stability condition in the form

$$
R_{L} \stackrel{!}{<} \sqrt{1+\left|L_{0}\right|^{2}-2\left|L_{0}\right| \cos \delta_{0}} .
$$

From the concept of the proposed empirical sine wave PID controller tuning method results, that the robust controller shifts the nominal point of the plant frequency response $G_{0}$ to the point $L_{0}$ of the unit circle at frequency $\omega_{n}$. Thus $\omega_{n}$ becomes amplitude crossover frequency for the open loop. As the point $L_{0}$ is lying on the circle $M_{1}$, the magnitude $\left|L_{0}\left(j \omega_{n}\right)\right|$ equals one

$$
\left|L_{0}\right|=\left|G_{0}\right|\left|G_{R}\right|=1
$$



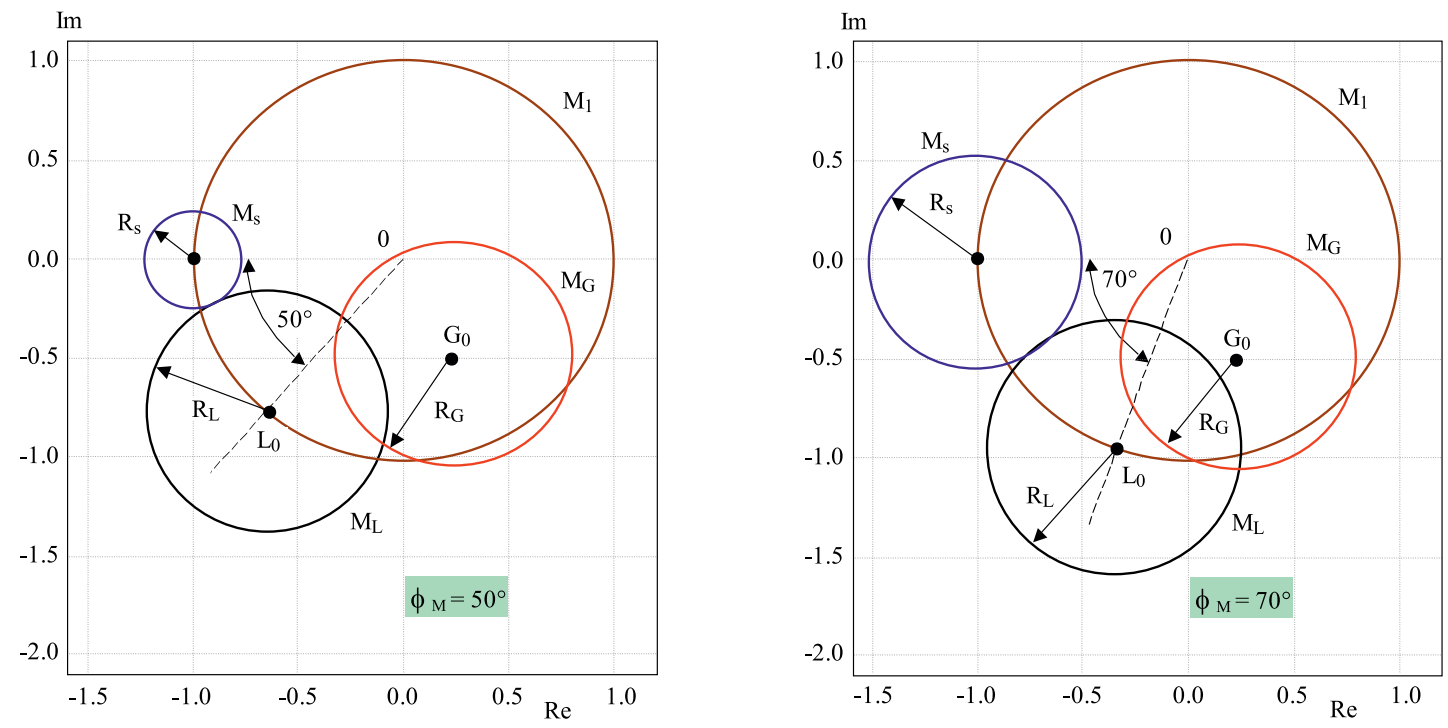

Fig. 8. Robust stability for different $\phi_{M}$

yielding the transformation ratio $\left|G_{R}\right|=\left|G_{0}\right|^{-1}$ between the radii $R_{G}$ and $R_{L}$ of the circles $M_{G}$ and $M_{L}$, respectively. The radius $R_{L}$ of the dispersion circle $M_{L}$ can be expressed as

$$
R_{L}=R_{G}\left|G_{R}\right|=R_{G}\left|G_{0}\right|^{-1} .
$$

Substituting (45) and (44) in (41) yields the robust stability condition in the following form

$$
R_{G}^{2}\left|G_{R}^{2}\right|^{-1} \stackrel{!}{<} 2-2 \cos \delta_{0}
$$

After minor manipulations, condition for calculating the angle $\delta_{0}$ is obtained

$$
\cos \delta_{0}<\left(1-\frac{R_{G}^{2}}{2\left|G_{0}^{2}\right|}\right) .
$$

According to the robust stability condition the chosen value $\delta_{0}$ is subsequently substituted into the phase condition (37) and subsequently parameters of the robust PID controller are calculated from (36) for $\delta_{0} \geq \phi_{M}$.

\section{VERIFICATION OF THE EMPIRICAL SINE WAVE METHOD ON A REAL PLANT}

The empirical sine wave method was applied for robust control of a physical model - the DC motor. In case of the DC motor the controlled output $y(t)$ is the speed, the input variable is the armature voltage generated by the control system implemented in Matlab-Realtime Workshop. To sense the output $y(t)$ a tachogenerator (TG) is used. The disturbance affecting the motor operation is the load torque $z(t)$, as depicted in the feedback control loop in Fig. 5.

Three identification experiments were carried out on the DC motor, for the minimum, medium and the maximum loads. Corresponding time responses are in Fig. 6.
Parameters of the designed robust PID controllers for two phase margin values $\phi_{M}=50^{\circ}$ and $70^{\circ}$ are in Table 1 .

Robust properties for both cases were verified by applying DC motor reference speed step change and after the transient response died out the load step change was applied. Corresponding time responses are in Fig. 7.

\section{CONCLUSION}

Stability and performance of designed robust control loops [5] have been proved by the time responses in Fig. 7 and by the position of the dispersion circle $M_{L}$ in Fig. 8 . Moreover, Figure 8 shows that the DC motor control loop designed for the phase margin $\phi_{M}=70^{\circ}$ determines a larger prohibited area in the complex plain delineated by the circle $M_{s}$ than the control-loop designed for a phase margin $\phi_{M}=50^{\circ}$. Both control loops have successfully coped with the step change of the motors load torque $z(t)$ occurred at $65 \mathrm{~s}$ with keeping a $100 \%$ steady-state accuracy.

\section{Acknowledgment}

This research work has been supported by the Scientific Grant Agency of the Ministry of Education of the Slovak Republic, Grant No. 1/0544/09.

\section{REFERENCES}

[1] ACKERMANN, J.: Robust Control System with Uncertain. Physical Parameters, Wiley\&Sons, Springer-Verlag, Berlin, London, New York, 1997, $3^{\text {rd }}$ printing.

[2] ÅstrÖM, K. J.-HÄGGLUND, T. : The Future of PID Control, IFAC Workshop on Digital Control PID00, Terrassa, Spain, April 5-7, 2000, 2000, pp. 19-30.

[3] DÚBRAVSKÁ, M.-HARSÁNYI, L. : Control of Uncertain Systems, J. Electrical Engineering 58 No. 4 (2007), 228-231. 
[4] ENDER, D. B.: Process Control Performance: Not as Good as You Think, Control Engineering 40 No. 10 (1993), 180-190.

[5] INGIMUNDARSON, A.—ÅSTRÖM, K. J.-HÄGGLUND, T. : Criteria for Design of PID Controllers, Department of Automatic Control, Lund, 2004.

[6] MORARI, M.-ZAFIRIOU, E.: Robust Processs Control, Prentice Hall, Englewood Cliffs, New Jersey, 1989.

[7] O'DWYER, A.: Handbook of PI and PID Controllers Tuning Rules, 2nd Edition, Imperial College Press, London, 2006.

[8] ROTAČ, V.: Avtomatizacija nastrojki system upravlenija, Energoatomizdat, Moskva, 1984. (in Russian)

[9] VESELÝ, V.: Easy Tuning of PID Controller, J. Electrical Engineering 54 No. 5-6 (2003), 136-139.

[10] VISOLI, A.: Practical PID Control, Springer-Verlag, London, 2006.

[11] YU, C.-C.: Autotuning of PID Controllers. A Relay Feedback Approach, 2nd Edition, Springer-Verlag, London, 2006.

[12] ZIEGLER, J. G.-NICHOLS, N. B.: Optimum Settings for Automatic Controllers, Trans. ASME 64 (2004), 759-768.

Recived 7 September 2009

Štefan Bucz (MSEE) was born in Komárno, Slovakia, in 1978. He graduated from the Faculty of Electrical Engineering and Information Technology, Slovak University of Technology, Bratislava, in 2005. At present he is a $\mathrm{PhD}$ student in the Institute of Control and Industrial Informatics, Faculty of Electrical Engineering and Information Technology. The main field of his study is robust control, tuning methods for industrial processes with unknown mathematical model and varying parameters.

Ladislav Marič (MSEE) was born in Dunajská Streda, Slovakia, in 1984. He graduated from the Faculty of Electrical Engineering and Information Technology, Slovak University of Technology, Bratislava, in 2009.

Ladislav Harsányi (Assoc Prof, PhD) was born in Ohrady, Slovakia, in 1936. He graduated from the Faculty of Electrical Engineering, Slovak University of Technology, Bratislava, in 1960. He obtained the PhD degree in Technical Cybernetics from the Faculty of Electrical Engineering of Slovak Technical University, in 1974. At present he is Associate Professor in the Institute of Control and Industrial Informatics, Faculty of Electrical Engineering and Information Technology. The main field of his research and teaching activities are theory of control, robust control, control of power systems and using computers in education.

Vojtech Veselý (Prof, DSc) was born in 1940. Since 1964 he has been with the Department of Automatic Control Systems at the Faculty of Electrical Engineering and Information Technology, Slovak University of Technology in Bratislava, where he has supervised $18 \mathrm{PhD}$ students up today. Since 1986 he has been Full Professor. His research interests include power system control, decentralized control, process control and optimization. He is author and co-author of more than 250 scientific papers.



EXPORT - IMPORT
EXPORT - IMPORT

of periodicals and of non-periodically printed matters, books and CD-ROMs

Krupinská 4 PO BOX 152, 85299 Bratislava 5, Slovakia tel: ++421 263839 472-3, fax: ++421 263839485 info@slovart-gtg.sk; http://www.slovart-gtg.sk



EXPORT - IMPORT 